\title{
The Visiting Medical Student Clerkship Program at Mayo Clinic
}

\author{
Paul S. Mueller, MD, MPH; Linda L. McConahey, BA; Laura J. Orvidas, MD; \\ SARAH M. Jenkins, MS; AND Mary J. Kasten, MD
}

\begin{abstract}
OBJECTIVE: To describe the history, objectives, statistics, and initiatives used to address challenges associated with the Mayo Clinic Visiting Medical Student (VMS) Clerkship Program.

MATERIALS AND METHODS: Mayo Clinic administrative records were reviewed for calendar years 1995 through 2008 to determine the effect of interventions to increase the numbers of appropriately qualified international VMSs and underrepresented minority VMSs. For numerical data, descriptive statistics were used; for comparisons, $\chi^{2}$ tests were performed.
\end{abstract}

RESULTS: During the specified period, 4908 VMSs participated in the Mayo VMS Program (yearly mean [SD], 351 [24]). Most students were from US medical schools (3247 [66\%]) and were male (3084 [63\%]). Overall, 3101 VMSs (63\%) applied for and 935 (30\%) were appointed to Mayo Clinic residency program positions. Interventions to address the challenge of large numbers of international students who participated in our VMS program but did not apply for Mayo residency positions resulted in significantly fewer international students participating in our VMS program $(P<.001)$, applying for Mayo residency program positions $(P<.001)$, and being appointed to residency positions $(P=.001)$. Interventions to address the challenge of low numbers of underrepresented minority students resulted in significantly more of these students participating in our VMS program $(P=.005)$, applying for Mayo residency positions $(P=.008)$, and being appointed to residency positions $(P=.04)$.

CONCLUSION: Our findings suggest that specific interventions can affect the characteristics of students who participate in VMS programs and who apply for and are appointed to residency program positions.

Mayo Clin Proc. 2010;85(8):723-727

TOEFL $=$ Test of English as a Foreign Language; USMLE $=$ US Medical Licensing Examination; VMS = visiting medical student

A visiting medical student (VMS) is a medical student who participates in an elective or clerkship at a medical school other than his or her own. Most US medical schools have VMS programs. ${ }^{1}$ About half of fourth-year US medical students participate in VMS electives and clerkships (also known as externships and audition electives). ${ }^{2-6}$ "Recruitment for residency programs" is the most frequently cited reason medical schools have VMS programs. ${ }^{7}$ However, published literature on VMS programs at specific US medical schools is minimal, particularly regarding the organization, challenges, and other aspects associated with running a VMS program.

Although Mayo Medical School was founded in 1972, ${ }^{8}$ Mayo Clinic has hosted VMSs since 1915. Currently, Mayo Clinic hosts approximately 350 VMSs each year at its
Minnesota, Arizona, and Florida campuses. ${ }^{9}$ Since its inception, the objectives of the Mayo Clinic VMS Clerkship Program have been as follows: (1) to offer VMSs a broad array of high-quality elective and clerkship experiences; (2) to offer Mayo Clinic faculty, fellows, and residents opportunities for teaching medical students; and (3) to recruit VMSs to Mayo's residency training programs..$^{9-11}$ Each year, about 30\% of Mayo Clinic's newly appointed residents are former Mayo VMS Program students.

Visiting medical students must be in the last quarter of their third year or in the fourth year of medical school and have completed 48 weeks of patient care. The duration of a visiting elective or clerkship is 1 month. Visiting medical students have the same clinical experiences and are evaluated in the same manner as Mayo Medical School students. Mayo Clinic does not charge tuition to VMSs (although international students pay an application fee).

During the past 10 years, the number of applicants to the Mayo VMS Program has grown. Because of this growth and limited clerkship and elective capacity to meet demand, not all students who apply to our VMS program are offered a clerkship or elective. However, we also observed that many VMSs, especially international students, had no intention of applying for or were not competitive in being appointed to Mayo Clinic residency program positions. Furthermore, Mayo Clinic leadership expressed a desire to increase the numbers of underrepresented minority VMSs who participate in our VMS program. We describe interventions that were implemented to address these challenges and the effects of these interventions.

\section{MATERIALS AND METHODS}

This study was deemed exempt from institutional review board evaluation as an education-related study under 45

From the Division of General Internal Medicine (P.S.M., M.J.K.), Mayo School of Graduate Medical Education (L.L.M.), Department of Otorhinolaryngology (L.J.O.), and Division of Biomedical Statistics and Informatics (S.M.J.), Mayo Clinic, Rochester, MN.

Dr Mueller is a member of the Boston Scientific Patient Safety Advisory Board and is an Associate Editor of Journal Watch General Medicine; neither of these activities is related to this article.

Individual reprints of this article are not available. Address correspondence to Paul S. Mueller, MD, MPH, Division of General Internal Medicine, Mayo Clinic, 200 First St SW, Rochester, MN 55905 (mueller.pauls@mayo.edu).

(ㄷ) 2010 Mayo Foundation for Medical Education and Research 
CFR 46.101(b)4. Data related to the Mayo Clinic VMS Clerkship Program were derived from Mayo Clinic administrative records and a computerized database. Data were available for 1995 through 2008 (data for VMSs from osteopathic medical schools were available for 1999 through 2008 only).

A US visiting medical student was defined as a visiting medical student from a US allopathic or osteopathic medical school, whereas an international visiting medical student was defined as a visiting medical student from a nonUS medical school. An underrepresented minority visiting medical student was defined as a visiting medical student who is a US citizen or permanent resident enrolled in a US Liaison Committee on Medical Education-accredited medical school and self-identified as black/African American, American Indian/Alaskan Native, Native Hawaiian/ US Pacific Islander, or Hispanic. ${ }^{12,13}$

\section{Interventions to Address Program Challenges}

We observed that many international VMSs who participated in our VMS program did not subsequently apply for Mayo residency program positions; this is a challenge given that a major objective of the Mayo VMS Program is residency program recruitment. Also, many international students lacked sufficient fluency in English. In response to these observations, international students, beginning in 2006, were required to successfully complete the US Medical Licensing Examination (USMLE) Step 1 and the Test of English as a Foreign Language (TOEFL). The rationale for this change was that international VMSs who had successfully completed USMLE Step 1 and TOEFL were more likely to pursue US residency program positions. Also, by reducing the number of elective and clerkship slots taken by VMSs who do not intend to apply for residency program positions, slots are available for students who do intend to apply for such positions. Finally, international VMSs who are fluent in English are more likely to have a positive elective or clerkship experience (as are their patients and faculty). Moreover, the application fee for international VMSs was increased from $\$ 250$ to $\$ 350$ to defray the costs associated with processing their applications.

Another challenge was low numbers of underrepresented minority students participating in our VMS program. To increase these numbers, beginning in 2004, the Mayo Clinic Office of Diversity began highlighting the Mayo VMS Program and a diversity scholarship (a competitive program provides $\$ 1000$ in financial support to underrepresented minority medical students who participate in the Mayo Clinic VMS Program ${ }^{9}$ ) in its annual Career Development Program for second- and third-year US underrepresented minority medical students. ${ }^{13}$ In addition, the numbers of visits by Mayo Clinic faculty to medical schools in the Upper Midwest with large numbers of underrepresented minority students were increased. The purpose of these visits is to recruit underrepresented minority students to participate in the Mayo VMS Program.

\section{Statistical Analyses}

Descriptive statistics were rounded to the nearest whole number. Between-group frequencies and percentages were compared using $\chi^{2}$. For comparisons, statistics for the years 2003 through 2005 vs the years 2006 through 2008 were used. These periods were selected on the basis of the timing of the interventions and when they would affect medical students participating in the Mayo VMS Program. GraphPad QuickCalcs ${ }^{14}$ (GraphPad Software, Inc, La Jolla, CA) was used. $P<.05$ was considered significant.

\section{RESULTS}

Overall Mayo Clinic VMS Clerkship Program Statistics During calendar years 1995 through 2008, the yearly mean (SD) number of applications received from potential VMSs was 585 (117). During the same period, the yearly average number of VMSs who actually participated in Mayo VMS Program electives and clerkships was 351 (24). Similar data regarding US, international, and underrepresented minority visiting students are found in Table 1 . Trends regarding the number of students who have participated in the program are graphically displayed in the Figure.

Most of the 4908 students (3247 [66\%]) who participated in the Mayo VMS Program were from US medical schools. During 1999 through 2008, the yearly mean number of VMSs from osteopathic schools was 35 (10). Most VMSs (3084 [63\%]) were men; this did not change when the data were stratified by country of origin. For example, during academic year 2007 through 2008, 192 (62\%) of the 312 US students and 37 (62\%) of the 60 international students were men.

\section{Appointment of Former VMSs to Mayo Clinic Residency Program Positions}

Of the 4908 VMSs who came to Mayo Clinic during 1995-2008, 3101 (63\%) subsequently applied for Mayo Clinic residency program positions (mean [SD] per year, 222 [23]), and of these, 935 (30\%) were appointed to Mayo residency program positions (mean per year, 67 [5]). Similar data for US, international, underrepresented minority, and nonunderrepresented minority visiting students are found in Table 1.

During 1995 through 2008, the percentage of international medical students who participated in the Mayo VMS Program and subsequently applied for Mayo residency program positions was significantly lower than the percentage 
TABLE 1. International vs US and URM vs Non-URM Visiting Medical Students Who Participated in Electives and Clerkships and Applied for and Were Appointed to Residency Positions at Mayo Clinic, 1995-2008

\begin{tabular}{|c|c|c|c|c|c|c|c|}
\hline \multirow[b]{2}{*}{ Visiting medical students } & \multirow[b]{2}{*}{ Overall } & \multicolumn{3}{|c|}{ By home country } & \multicolumn{3}{|c|}{ By minority status } \\
\hline & & International & US & $P$ value & URM & Non-URM & $P$ value \\
\hline \multicolumn{8}{|l|}{$\begin{array}{l}\text { Participated in electives and } \\
\text { clerkships at Mayo }\end{array}$} \\
\hline No. & 4908 & 1661 & 3247 & NA & 410 & 2837 & NA \\
\hline Mean No. (SD) per year & $351(24)$ & $119(35)$ & $232(33)$ & & $29(8)$ & $202(31)$ & \\
\hline \multicolumn{8}{|l|}{$\begin{array}{l}\text { Applied for a Mayo residency } \\
\text { program position }\end{array}$} \\
\hline No. $(\%)$ & $3101(63)$ & $334(20)$ & $2767(85)$ & $<.001$ & $260(63)$ & $2507(88)$ & $<.001$ \\
\hline Mean No. (SD) per year & $222(23)$ & $24(9)$ & $198(22)$ & & $19(6)$ & $179(21)$ & \\
\hline \multicolumn{8}{|c|}{ Applied for and were appointed to a } \\
\hline \multicolumn{8}{|c|}{ Mayo residency program position } \\
\hline No. $(\%)$ & $935(30)$ & $110(33)$ & $825(30)$ & .23 & $87(33)$ & 738 (29) & .18 \\
\hline Mean No. (SD) per year & $67(5)$ & $8(4)$ & $59(5)$ & & $6(2)$ & $53(4)$ & \\
\hline
\end{tabular}

$\mathrm{NA}=$ not applicable; URM = underrepresented minority.

of US medical students (20\% vs $85 \% ; P<.001)$. However, the percentage of international vs US VMSs who applied for and were appointed to Mayo residency program positions did not differ (33\% vs 30\%; $P=.23$ ) (Table 1).

The percentage of underrepresented minority VMSs who participated in the Mayo VMS Program and applied for Mayo residency program positions was significantly lower than the percentage of non-underrepresented minority students $(63 \%$ vs $88 \% ; P<.001)$. However, the percentage of underrepresented minority vs non-underrepresented minority VMSs who applied for and were appointed to Mayo residency program positions did not differ (33\% vs 29\%; $P=.18$ ) (Table 1).

Notably, from 1995 through 2008, a total of 560 students attended Mayo Medical School (mean [SD] per year, 40 [4]). Of these, 431 (77\%) applied for Mayo residency program positions (mean per year, 31 [6]), 219 (51\%) of whom were appointed to Mayo residency program positions (mean per year, 16 [3]). Compared with VMSs, Mayo Medical School students were significantly more likely to be appointed to Mayo residency program positions (30\% vs $51 \% ; P<.001)$.

\section{EfFEcts OF InTERVENTIONS}

After implementing the previously described interventions, we observed significant changes in the numbers of international and US VMSs participating in the Mayo VMS Program and later appointed to Mayo Clinic residency program positions. When comparing the data for the 3 years before and the 3 years after requiring successful completion of USMLE Step 1 and TOEFL and the increased application fee, the percentage of international students who applied to the Mayo VMS Program decreased from 20032005 to $2006-2008$ (49\% vs $17 \%$ ), and the percentage of US students correspondingly increased significantly from 2003-2005 to $2006-2008$ (51\% vs $83 \% ; P<.001)$. Similar results were observed for percentages of US vs international students who did electives and clerkships at Mayo, applied for Mayo residency program positions, and were appointed to Mayo residency program positions (Table 2). Despite these changes, the percentage of US vs international VMSs who applied for and were appointed to Mayo residency program positions during 2006-2008 did not differ significantly ( $31 \%$ vs $35 \% ; P=.57)$.

We also observed changes in the numbers of underrepresented vs non-underrepresented minority VMSs participating in the Mayo VMS Program. The percentage of underrepresented minority VMSs increased from 2003-2005 to

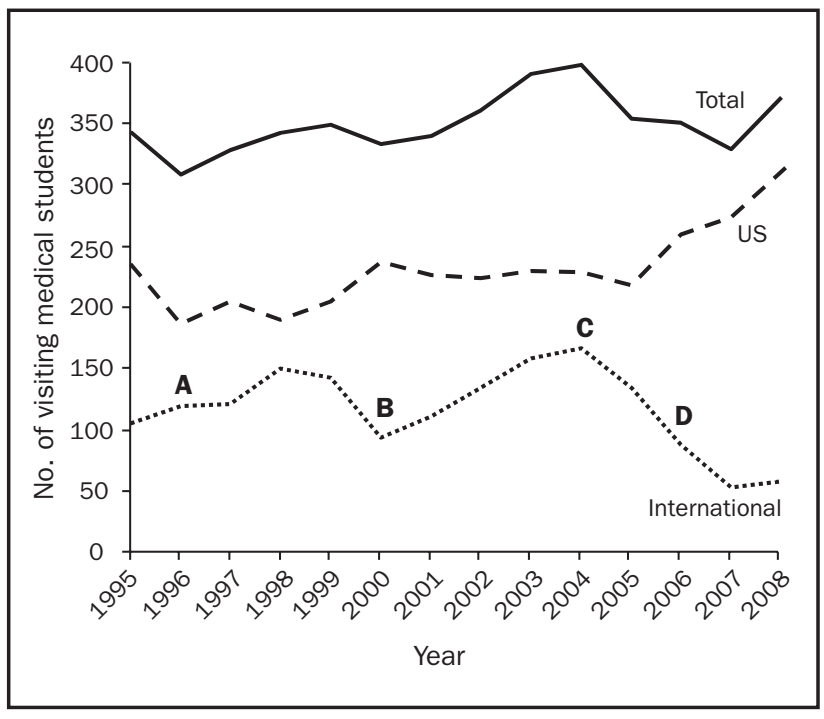

FIGURE. Total, US, and international visiting medical students (VMSs) at Mayo Clinic and interventions implemented (1995-2008). A, $\$ 50$ application fee for international VMSs. B, \$100 application fee for international VMSs; C, \$250 application fee for international VMSs; D, \$350 application fee for international VMSs and successful completion of US Medical Licensing Examination Step 1 and Test of English as a Foreign Language. 
TABLE 2. International vs US Visiting Medical Students Who Applied for and Did Electives and Clerkships at Mayo and Subsequently Applied for and Were Appointed to Residency Positions at Mayo Clinic, 2003-2005 vs 2006-2008

\begin{tabular}{|c|c|c|c|}
\hline Student status & $2003-2005$ & 2006-2008 & $P$ value \\
\hline \multicolumn{4}{|c|}{$\begin{array}{l}\text { Visiting medical student } \\
\text { applications }\end{array}$} \\
\hline US & $1053(51)$ & $1199(83)$ & $<.001$ \\
\hline International & $994(49)$ & $250(17)$ & \\
\hline \multicolumn{4}{|c|}{ Visiting medical students } \\
\hline US & $680(59)$ & $848(81)$ & $<.001$ \\
\hline International & $464(41)$ & $205(20)$ & \\
\hline \multicolumn{4}{|c|}{$\begin{array}{l}\text { Visiting medical students } \\
\text { who applied for a Mayo } \\
\text { residency position }\end{array}$} \\
\hline US & $540(87)$ & $611(95)$ & $<.001$ \\
\hline International & $82(13)$ & $34(5)$ & \\
\hline \multicolumn{4}{|c|}{$\begin{array}{l}\text { Visiting medical students } \\
\text { appointed to a Mayo } \\
\text { residency position }\end{array}$} \\
\hline US & $168(84)$ & $190(94)$ & .001 \\
\hline International & $32(16)$ & $12(6)$ & \\
\hline
\end{tabular}

Values are number (percentage), unless indicated otherwise. Percentages may not total $100 \%$ due to rounding.

2006-2008 (5\% vs 13\%), and the percentage of non-underrepresented minority students correspondingly decreased from $2003-2005$ to $2006-2008$ (95\% vs $87 \%)(P<.001)$. Similar results were observed for the percentages of underrepresented minority vs non-underrepresented minority students who participated in electives and clerkships at Mayo Clinic, applied for Mayo residency program positions, and were appointed to Mayo residency program positions (Table 3 ). Despite these changes, the percentage of underrepresented minority vs non-underrepresented minority VMSs who applied for and were appointed to Mayo residency program positions during 2006-2008 did not differ significantly (36\% vs $31 \% ; P=.39$ ).

TABLE 3. URM vs Non-URM Visiting Medical Students Who Applied for and Did Electives and Clerkships at Mayo and Subsequently Applied for and Were Appointed to Residency Positions at Mayo Clinic, 2003-2005 vs 2006-2008

\begin{tabular}{lccc}
\hline \multicolumn{1}{c}{ Student status } & $2003-2005$ & $2006-2008$ & $P$ value \\
\hline Visiting medical students & & & \\
$\quad$ URM & $59(5)$ & $112(13)$ & .005 \\
$\quad$ Non-URM & $1085(95)$ & $941(87)$ & \\
Visiting medical students & & & \\
$\quad$ who applied for a Mayo & & & \\
residency position & $33(5)$ & $64(10)$ & .008 \\
$\quad$ URM & $589(95)$ & $581(90)$ & \\
$\quad$ Non-URM & & & \\
Visiting medical students & & & \\
appointed to a Mayo & & & \\
residency position & $10(5)$ & $23(12)$ & .04 \\
$\quad$ URM & $190(95)$ & $179(88)$ & \\
$\quad$ Non-URM &
\end{tabular}

Values are number (percentage), unless indicated otherwise. URM $=$ underrepresented minority.
Notably, although we observed differences in the distributions of international vs US and underrepresented minority vs non-underrepresented minority students before and after the interventions (Tables 2 and 3), stratifying our analysis by period (pre- vs post-interventions) did not affect our overall conclusions (data not shown). In other words, any differences (or lack thereof) observed between international vs US and underrepresented minority vs nonunderrepresented minority students were similar within each period.

\section{DISCUSSION}

To our knowledge, this is the first report that describes the history, objectives, statistics, and interventions used to address challenges associated with a large VMS program. We found that international VMSs were less likely than US students to apply for Mayo residency program positions. This finding suggested that many international students participate in our VMS program for reasons other than securing a Mayo residency program position (eg, acquire novel learning and travel experiences). Nevertheless, a major objective of our VMS program is recruitment to our residency training programs. Hence, we sought to reduce the number of international VMSs who had no intention of applying for or were not competitive in being appointed to Mayo Clinic residency program positions. The requirement of successful completion of the USMLE Step 1 and TOEFL for international VMSs likely achieved this end. Needless to say, international students who take the USMLE Step 1 and are fluent in English are more likely to pursue residency program positions in the United States than international students who do not successfully complete these examinations. At the same time, the application fee for international students was increased from $\$ 250$ to $\$ 350$; we think this intervention had minimal impact, particularly given that prior fee increases had little impact (Figure). Currently, despite lower numbers of international medical students who participate in our VMS program, those who apply for Mayo residency program positions are just as likely as US students to be appointed to Mayo residency program positions.

We also addressed the challenge of low numbers of underrepresented minority VMSs who participated in our VMS program. This finding suggested that underrepresented minority medical students have more barriers (eg, costs) than non-underrepresented minority medical students to participating in our VMS program or may not regard participating in our VMS program as highly as do nonuderrepresented minority students. Nevertheless, we found that intentional efforts, especially our Career Development Program and visits by our faculty to medical schools that 
have large numbers of underrepresented minority students (for the purpose of recruiting these students to participate in our VMS program), resulted in significantly more underrepresented minority medical students participating in the Mayo VMS Program and applying for and being appointed to Mayo Clinic residency program positions.

Our findings also validate the importance of the Mayo VMS Program for recruitment to Mayo residency training program positions. Although Mayo Medical School students were significantly more likely to be appointed to Mayo Clinic residency program positions, the school is too small (about 50 students per class circa 2010) to provide sufficient numbers of candidates to fill Mayo residency program positions. For example, of the 287 newly appointed residents at Mayo Clinic during the 2008-2009 academic year, 67 (23\%) were former Mayo VMS Program VMSs, whereas only 15 (5\%) were former Mayo Medical School students.

Our analysis has several limitations. It is a retrospective review of the before-and-after effects of interventions that were neither randomized nor blinded. Also, the analysis represents the findings at a single institution, and generalizing the results to other institutions that host VMSs may not be possible.

\section{CONCLUSION}

Our findings suggest that specific interventions can affect the characteristics of students who participate in VMS programs and who apply for and are appointed to residency program positions. Individuals who direct or coordinate VMS programs elsewhere may find this report informative.

\section{REFERENCES}

1. Association of American Medical Colleges. Extramural electives compendium. Tomorrow's Doctors Web site. www.aamc.org/students/medstudents /electives/start.htm. Accessed March 13, 2009

2. Association of American Medical Colleges. 1991 Graduating Student Survey Results: All Schools Summary. Washington, DC: Association of American Medical Colleges Section of Educational Research; 1991.

3. Association of American Medical Colleges. 1992 Graduating Student Survey Results: All Schools Summary. Washington, DC: Association of American Medical Colleges Section for Educational Research; 1992.

4. Association of American Medical Colleges. 1993 Medical School Graduation Questionnaire: Summary Report for All Schools. Washington, DC: Association of American Medical Colleges Section for Educational Research; 1993.

5. Association of American Medical Colleges. 1994 Medical School Graduation Questionnaire: Summary Report for All Schools. Washington, DC: Association of American Medical Colleges Section for Educational Research; 1994.

6. Association of American Medical Colleges. 1995 Medical School Graduation Questionnaire Survey Results: All Schools Summary. Washington, DC: Association of American Medical Colleges Section for Educational Research; 1995.

7. Mueller PS, McConahey LL, Orvidas LJ, Lee MC, Bowen JM, Beckman TJ, Kasten MJ. Visiting medical student elective and clerkship programs: a survey of US and Puerto Rico allopathic medical schools [published online ahead of print June 7, 2010]. BMC Med Educ. 2010;10:41. doi:10.1186/1472-6920-10-41.

8. Mayo Clinic, Mayo School of Graduate Medical Education, College of Medicine. History. www.mayo.edu.msgme/history.html. Accessed March 13, 2009.

9. Mayo Clinic. Mayo School of Graduate Medical Education; College of Medicine. Visiting Medical Student Clerkship Program. www.mayo.edu /msgme/clerkships.html. Accessed March 13, 2009.

10. Clinic experience is "rewarding": 23 medical students are "clerks." Mayovox. 1963;14(19): 1 .

11. Telling, teaching, recruiting the best and brightest: Visiting Medical Student Clerkship Program. Mayo Alumni. 2008 Spring;3-7.

12. Department of Education. Proposed guidance on maintaining, collecting, and reporting data on race and ethnicity to the U.S. Department of Education. Federal Register. 2006;71(151):44866. http://frwebgate.access.gpo.gov /cgi-bin/getpage.cgi?dbname=2006_register \&position=all\&page=44866. Accessed June 8, 2010.

13. Mayo Clinic. Mayo School of Graduate Medical Education; College of Medicine. Career Development Program. http://www.mayo.edu/msgme /diversity-cdc.html. Accessed February 23, 2010.

14. GraphPad Software. QuickCalcs: online calculators for scientists. http://graphpad.com/quickcalcs/index.cfm. Accessed June 8, 2010. 